This item was submitted to Loughborough's Research Repository by the author.

Items in Figshare are protected by copyright, with all rights reserved, unless otherwise indicated.

\title{
Higher-order gradient continuum modelling of periodic lattice materials
}

PLEASE CITE THE PUBLISHED VERSION

http://dx.doi.org/10.1016/j.commatsci.2011.05.025

PUBLISHER

(c) Elsevier

VERSION

AM (Accepted Manuscript)

LICENCE

CC BY-NC-ND 4.0

REPOSITORY RECORD

Lombardo, Mariateresa, and Harm Askes. 2019. "Higher-order Gradient Continuum Modelling of Periodic Lattice Materials". figshare. https://hdl.handle.net/2134/11450. 
This item was submitted to Loughborough's Institutional Repository (https://dspace.lboro.ac.uk/) by the author and is made available under the following Creative Commons Licence conditions.

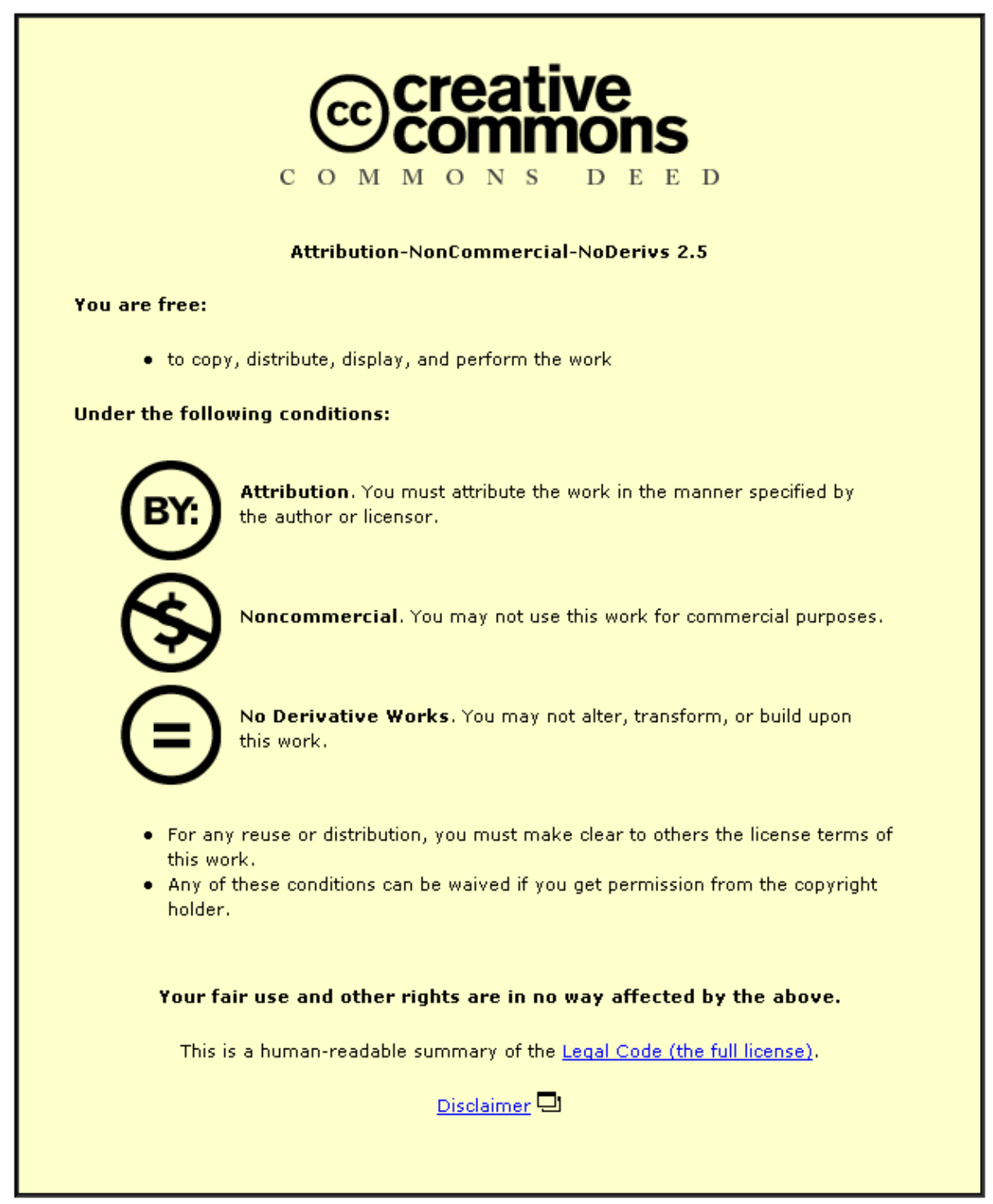

For the full text of this licence, please go to: http://creativecommons.org/licenses/by-nc-nd/2.5/ 


\title{
Higher-order gradient continuum modelling of periodic lattice materials
}

\author{
Mariateresa Lombardo ${ }^{\mathrm{a}, *}$, Harm Askes $^{\mathrm{a}}$ \\ ${ }^{a}$ Department of Civil and Structural Engineering, University of Sheffield, Sir Frederick Mappin Building, Sheffield S1 3JD, United Kingdom
}

\begin{abstract}
The dynamic behaviour of periodic lattice materials is investigated using an equivalent higher-order continuum model obtained by homogenisation of the equations of motion. A gradient continuum enriched with higher-order inertia terms is developed using a combination of finite element discretisation of the unit cell and the continualisation approach. The analysis of the dispersion relations shows that the proposed model is able to capture correctly the physical phenomenon of wave dispersion in lattice structures which is overlooked by classical continuum theories.
\end{abstract}

Keywords: periodic lattice, higher-order continuum, continualisation, wave propagation, dispersion relations

\section{Introduction}

In recent years, new classes of cellular solids and lattice materials have been used for a wide range of engineering applications, such as light-weight structures, vibration control devices or energy absorption systems, since it is well known that latticelike materials enhance the static and dynamic responses compared to their solid counterpart. The improvement of the properties depends on the material from which they are made, their relative density, and their internal geometrical structure [1].

In general, periodic lattice structures can be considered as models of many systems that are simplified as assemblies of beam elements rigidly connected or joined through hinges, organised according to a given topology. The dynamic behaviour of periodic structures has attracted the interest of many researchers. The wave propagation characteristics measured using non-destructive techniques allow to obtain important information regarding the materials under investigation. The employ of analytical methods to represent the dynamic behaviour of many cellular and lattice material has been the subject of a number of studies [2, 3].

A realistic representation of the dynamics of periodic solids would require modelling the microstructure with a large number of elements, resulting in very large number of degrees of freedom. Since such discrete analysis is computationally expensive, it is convenient to represent the cellular solid on the macroscale as an equivalent homogeneous material while at the lower level it is modelled by means of a unit cell (UC) considered as a rigid-joint network of beams. The homogenisation techniques provide governing equations at macroscopic level which contain information about the microstructure. Unfortunately, models based on classical elasticity theory do not give realistic predictions of many microstructural effects as for ex-

\footnotetext{
${ }^{*}$ Corresponding author

Email address: m. lombardo@sheffield.ac.uk (Mariateresa Lombardo)
}

ample the wave dispersion. This phenomenon is important because it governs the change of shape of a pulse as it propagates through a medium.

The intent of the present work is to propose a higher-order continuum model approximating the periodic lattice material that can capture the dispersion of elastic waves and whose implementation in commercial finite elements is relatively simple. The approach adopted to obtain the equivalent continuum differential equations is based on standard finite element (FE) description of the UC in conjunction with the continualisation technique derived from the Taylor series expansion of the kinematic variables. Higher-order continuum models enriched by higher-order terms are obtained accounting for the microstructural length in the macrostructural material model, see [4] for an extended list of references. Finally, the dispersion relations for the discrete system and the proposed continuum approximations have been used to quantify the frequency range of validity of the proposed higher-order model.

\section{General procedure of continuum modelling}

In this section, to illustrate the adopted homogenisation approach, a material with square lattice microstructure is chosen for simplicity even though the technique can be easily generalised for complex structures with generic topology. The rigidjoint network of beams represents the computational scheme used for the material with underlying microstructure of the kind shown in figure 1.

The procedure that leads to the continuum approximation is founded on standard FE discretisation of the UC along with the continualisation technique based on the Taylor series expansion of the variable fields [5].

The continuum modelling procedure can be summarised as follows:

- a representative UC is identified from the material with periodic lattice microstructure and the degrees of freedom 
(DOFs) for each joint of the unit cell are considered as nodes as in conventional FE analysis;

- the interactions between joints are defined from the structural properties of the beams connecting the nodes;

- the discrete equations of motion for the UC are evaluated assembling the mass and stiffness matrices of the constituents following a standard finite element approach;

- the equations of motion for the node at the centre of the UC (reference node) are found in terms of displacements and rotations of the reference and neighbouring joints;

- the continuum approximation is obtained by introducing continuous displacement fields and by expressing the neighbour displacements and rotations in terms of the displacements and rotations of the reference node using a Taylor series expansion.

The macrostructural continuum model finally obtained is enriched by higher-order terms accounting for the internal length that is dependent on the dimension of the underlying microstructure. As a result of the order of approximation in the Taylor series expansion, different forms of the homogenised equations of motion can be derived. In order to study the performance of the different models, the analysis of the dispersion curves will be analysed.

\subsection{Finite element modelling of the unit cell: in-plane be- haviour of a square lattice}

Following the general approach described in the previous section, we focus our attention on the material whose unit cell is described by a square lattice of beams such that the homogenised material results in a special case of an orthotropic continuum.

The unit cell of the lattice is modelled as a rigid-joint network of four Euler-Bernoulli beams of uniform section $A$, length $\ell$, material density $\rho$ and Young's modulus $E$. The kinematics of each beam is described by two in-plane translational degrees of freedom (horizontal $u_{i}$ and vertical $v_{i}$ displacements ) and one rotation $\theta_{i}$ about the out-of-plane axes at each node, according to figure 1 .

The element mass and stiffness matrices are formulated in the element local reference system. Derivation of the element mass and stiffness matrices in the global reference system can be achieved by the use of the transformation matrix $\boldsymbol{T}$

$$
\boldsymbol{T}=\left[\begin{array}{cccccc}
\cos (\alpha) & \sin (\alpha) & 0 & 0 & 0 & 0 \\
-\sin (\alpha) & \cos (\alpha) & 0 & 0 & 0 & 0 \\
0 & 0 & 1 & 0 & 0 & 0 \\
0 & 0 & 0 & \cos (\alpha) & \sin (\alpha) & 0 \\
0 & 0 & 0 & -\sin (\alpha) & \cos (\alpha) & 0 \\
0 & 0 & 0 & 0 & 0 & 1
\end{array}\right]
$$

where $\alpha$ is the angle between the local axis and the global axis. The stiffness and mass element matrices can then be written as

$$
\begin{gathered}
\boldsymbol{K}_{g}^{(e)}=\boldsymbol{T}^{T} \boldsymbol{K}^{(e)} \boldsymbol{T} \\
\boldsymbol{M}_{g}^{(e)}=\boldsymbol{T}^{T} \boldsymbol{M}^{(e)} \boldsymbol{T}
\end{gathered}
$$

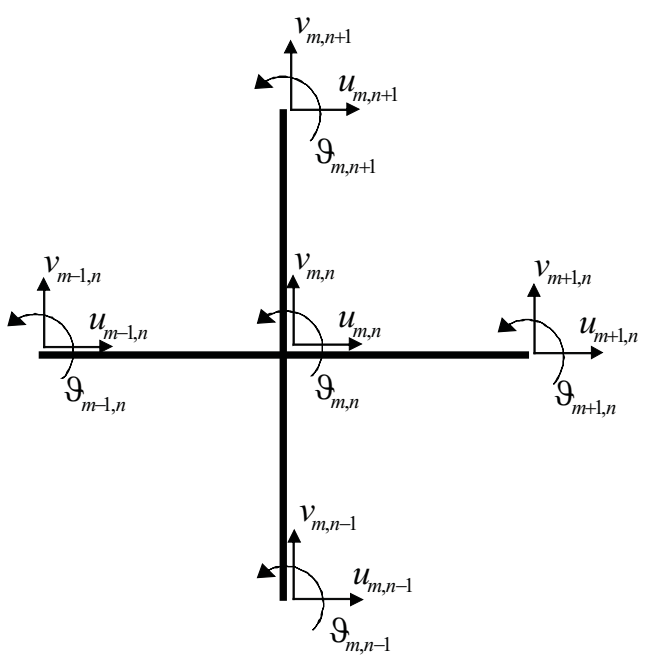

Figure 1: Unit cell

In equation $2 \mathrm{a}, \boldsymbol{K}^{(e)}$ denotes the element stiffness matrices that is

$$
\boldsymbol{K}^{(e)}=\left[\begin{array}{cccccc}
\frac{E A}{\ell} & 0 & 0 & -\frac{E A}{\ell} & 0 & 0 \\
0 & \frac{12 E I}{\ell^{3}} & \frac{6 E I}{\ell^{2}} & 0 & -\frac{12 E I}{\ell^{3}} & \frac{6 E I}{\ell^{2}} \\
0 & \frac{6 E I}{\ell^{2}} & \frac{4 E I}{\ell} & 0 & -\frac{6 E I}{\ell^{2}} & \frac{2 E I}{\ell} \\
-\frac{E A}{\ell} & 0 & 0 & \frac{E A}{\ell} & 0 & 0 \\
0 & -\frac{12 E I}{\ell^{3}} & -\frac{6 E I}{\ell^{2}} & 0 & \frac{12 E I}{\ell^{3}} & -\frac{6 E I}{\ell^{2}} \\
0 & \frac{6 E I}{\ell^{2}} & \frac{2 E I}{\ell} & 0 & -\frac{6 E I}{\ell^{2}} & \frac{4 E I}{\ell}
\end{array}\right]
$$

where $A=h^{2}$ is the cross-sectional area and $I=\frac{1}{12} h^{4}$ is the second moment of area.

In equation $2 \mathrm{~b} \boldsymbol{M}^{(e)}$ is the mass matrix of a beam that can be assumed in its lumped form as follows

$$
\boldsymbol{M}^{(e)}=\frac{\rho A \ell}{2} \operatorname{diag}\{1,1,0,1,1,0\} .
$$

After assembling the elements in global reference, the equation of motion for the unit cell can be expressed in matrix form as

$$
K d+M \ddot{d}=\mathbf{0}
$$

The vector $\boldsymbol{d}$ contains the displacements (translations and rotations) as shown in figure 1 . The dynamic behaviour of the unit cell with frame structure can be described by means of the equations of motion of the reference node $(m, n)$ common to the four beams.

\subsection{Discretised equations of motion and derivation of contin- uum equations}

To derive the continuum equations, we consider the three equations from the system (5) describing the motion of the 
node $(m, n)$. The equations have the form

$$
\begin{aligned}
& 2 \ell^{2} \frac{\rho}{E} \ddot{u}_{m, n}+2\left(1+\beta^{2}\right) u_{m, n}-u_{m+1, n}-u_{m-1, n} \\
&- \beta^{2}\left(u_{m, n-1}+u_{m, n+1}\right)+\frac{1}{2}\left(\theta_{m, n-1}-\theta_{m, n+1}\right)=0 \\
& 2 \ell^{2} \frac{\rho}{E} \ddot{v}_{m, n}+2\left(1+\beta^{2}\right) v_{m, n}-v_{m, n+1}-v_{m, n-1} \\
&-\beta^{2}\left(v_{m+1, n}+v_{m-1, n}\right)+\frac{1}{2}\left(\theta_{m+1, n}-\theta_{m-1, n}\right)=0 \\
& u_{m, n+1}-u_{m, n-1}+v_{m-1, n}-v_{m+1, n} \\
&+\frac{1}{3} \ell\left(\theta_{m-1, n}+\theta_{m, n-1}+\theta_{m, n+1}+\theta_{m+1, n}+8 \theta_{m, n}\right)=0
\end{aligned}
$$

where a dot represents a derivative with respect to time and DoFs numbering is given in figure 1 . The continualisation technique consists in replacing the vector of generalised discrete displacements $\boldsymbol{q}_{m, n}(t)=\left\{u_{m, n}(t), v_{m, n}(t), \theta_{m, n}(t)\right\}^{\mathrm{T}}$ with the vector of continuous components $\boldsymbol{q}(x, y ; t)=$ $\{u(x, y ; t), v(x, y ; t), \theta(x, y ; t)\}^{T}$.

For the discrete DoFs of the neighbouring lattice points, Taylor series expansions are used, that is

$$
\boldsymbol{q}_{m \pm 1, n \pm 1}(t)=\boldsymbol{q}(x \pm \ell, y \pm \ell ; t)=\sum_{i=0}^{N} \sum_{j=0}^{N} \frac{( \pm \ell)^{i}}{i !} \frac{( \pm \ell)^{j}}{j !} \frac{\partial^{i+j} \boldsymbol{q}(x, y ; t)}{\partial x^{i} \partial y^{j}}
$$

where $\boldsymbol{q}=\{u(x, y ; t), v(x, y ; t), \theta(x, y ; t)\}^{\mathrm{T}}$ is the vector of continuous generalised displacements.

By using the Taylor series expansion (7) in the discrete equations (6), keeping derivatives up to the second order, the system of equations is

$$
\begin{aligned}
& 2 \rho \ddot{u}-E\left(u_{x x}+\beta^{2} u_{y y}+\beta^{2} \theta_{y}\right)=0 \\
& 2 \rho \ddot{v}-E\left(\beta^{2} v_{x x}+v_{y y}-\beta^{2} \theta_{x}\right)=0 \\
& 2 \theta+u_{y}-v_{x}+\frac{1}{6} \ell^{2}\left(\theta_{x x}+\theta_{y y}\right)=0
\end{aligned}
$$

where $\beta=h / \ell$ is the geometric parameter that represents the slenderness of the beams. The subscripts $x$ and $y$ denote the spatial derivatives with respect to the spatial coordinates $x$ and $y$.

The equations (8), often called equations of micropolar elasticity [6-8], differ from the equations of classical theory elasticity by the additional equation of the microrotational wave. Neglecting the terms proportional to $\ell^{2}$ in the third expression of (8) to eliminate the rotational DoF $\theta$ from the system yields to the equations of conventional two-dimensional elasticity

$$
\begin{aligned}
& 2 \rho \ddot{u}=\frac{1}{2} E\left(2 u_{x x}+\beta^{2} u_{y y}+\beta^{2} v_{x y}\right) \\
& 2 \rho \ddot{v}=\frac{1}{2} E\left(\beta^{2} v_{x x}+2 v_{y y}+\beta^{2} u_{x y}\right)
\end{aligned}
$$

If derivatives up to the fourth order are kept in expression (7), the equations of motion take the form

$$
\begin{aligned}
& 2 \rho \ddot{u}=E\left(u_{x x}+\beta^{2} u_{y y}+\beta^{2} \theta_{y}\right)+\frac{1}{12} E \ell^{2}\left(u_{x x x x}+\beta^{2} u_{y y y y}+2 \beta^{2} \theta_{y y y}\right) \\
& 2 \rho \ddot{v}=E\left(\beta^{2} v_{x x}+v_{y y}-\beta^{2} \theta_{x}\right)+\frac{1}{12} E \ell^{2}\left(v_{y y y y}+\beta^{2} v_{x x x x}-2 \beta^{2} \theta_{x x x}\right) \\
& 2 \theta=v_{x}-u_{y}-\frac{1}{6} \ell^{2}\left(\theta_{x x}+\theta_{y y}-v_{x x x}+u_{y y y}\right)-\frac{1}{72} \ell^{4}\left(\theta_{x x x x}+\theta_{y y y y}\right)
\end{aligned}
$$

The rotational DoF $\theta$ can be again eliminated from the system (10) and neglecting the terms proportional to $\ell^{4}$ the equations of motion reduce to

$$
\begin{array}{r}
2 \rho\left[\ddot{u}+\frac{1}{12} \ell^{2}\left(\ddot{u}_{y y}-\ddot{v}_{x y}\right)\right]=\frac{1}{2} E\left(2 u_{x x}+\beta^{2} u_{y y}+\beta^{2} v_{x y}\right) \\
+\frac{1}{12} E \ell^{2}\left[u_{x x x x}+u_{x x y y}-\left(1-\beta^{2}\right) v_{x y y y}\right] \\
2 \rho\left[\ddot{v}+\frac{1}{12} \ell^{2}\left(\ddot{v}_{x x}-\ddot{u}_{x y}\right)\right]=\frac{1}{2} E\left(2 v_{y y}+\beta^{2} v_{x x}+\beta^{2} u_{x y}\right) \\
+\frac{1}{12} E \ell^{2}\left[v_{y y y y}+v_{x x y y}-\left(1-\beta^{2}\right) u_{x x y y}\right]
\end{array}
$$

The equations (11) differ from equations of classical elasticity (9) by the higher gradient inertia and stiffness terms. Because the considered continuum approximation features higher order spatial derivatives, the application of this model to finite domains would require formulating appropriate boundary conditions. Additionally, the study of the dispersion relations of theory (11) shows an anomalous behaviour associated with the higher order gradients entering in the governing equations [9].

It appears to be beneficial to consider theories that contain higher order inertia terms only, see Ref. [10]. To this end, the continuum model (11) can be generalised to model the dispersion phenomenon along with stability. A way to deal with the problem consists in constructing composite equations from (11) and (9), then identify the terms with higher order spatial derivatives that can be neglected and retain the higher order inertia terms . In our case we consider the superpositions $\left\{(11 a)+C_{1} \ell^{2}(9 a)_{x x}+C_{2} \ell^{2}(9 a)_{y y}+C_{3} \ell^{2}(9 b)_{x y}\right\}$ and $\left\{(11 b)+C_{4} \ell^{2}(9 b)_{x x}+C_{5} \ell^{2}(9 b)_{y y}+C_{6} \ell^{2}(9 a)_{x y}\right\}$. This yields

$$
\begin{aligned}
2 \rho \ddot{u} & +\frac{1}{6} \rho \ell^{2}\left[12 C_{1} \ddot{u}_{x x}+\left(1+12 C_{2}\right) \ddot{u}_{y y}+\left(12 C_{3}-1\right) \ddot{v}_{x y}\right]= \\
& \frac{1}{2} E\left(2 u_{x x}+\beta^{2} u_{y y}+\beta^{2} v_{x y}\right)+\frac{1}{2} E \ell^{2}\left[\left(2 C_{1}+\frac{1}{6}\right) u_{x x x x}\right. \\
& +\left(C_{1} \beta^{2}+2 C_{2}+C_{3} \beta^{2}+\frac{1}{6}\right) u_{x x y y}+C_{2} \beta^{2} u_{y y y y} \\
& \left.+\left(C_{2} \beta^{2}+2 C_{3}-\frac{1}{6}\left(1-\beta^{2}\right)\right) v_{x y y y}+\beta^{2}\left(C_{1}+C_{3}\right) v_{x x x y}\right]
\end{aligned}
$$

$$
\begin{aligned}
2 \rho \ddot{v} & +\frac{1}{6} \rho \ell^{2}\left[\left(1+12 C_{4}\right) \ddot{v}_{x x}+12 C_{5} \ddot{v}_{y y}+\left(12 C_{6}-1\right) \ddot{u}_{x y}\right]= \\
& \frac{1}{2} E\left(2 v_{y y}+\beta^{2} v_{x x}+\beta^{2} u_{x y}\right)+\frac{1}{12} E \ell^{2}\left[\left(2 C_{5}+\frac{1}{6}\right) v_{y y y y}\right. \\
& +\left(2 C_{4}+\beta^{2} C_{5}+\beta^{2} C_{6}+\frac{1}{6}\right) v_{x x y y}+C_{4} \beta^{2} v_{x x x x} \\
& \left.+\left(\beta^{2} C_{4}+2 C_{6}-\frac{1}{6}\left(1-\beta^{2}\right)\right) u_{x x x y}+\beta^{2}\left(C_{5}+C_{6}\right) u_{x y y y}\right]
\end{aligned}
$$

where $C_{i}(i=1, \ldots, 6)$ are arbitrary parameters. A theory with only inertia terms requires vanishing of all corresponding coefficients of the ten different fourth-order spatial derivative terms in the governing equations (12a) and (12b). Unfortunately, the 


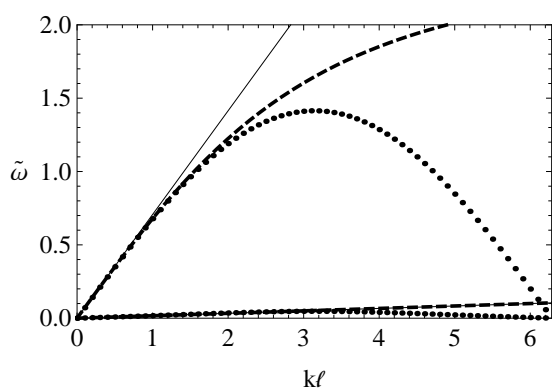

(a)

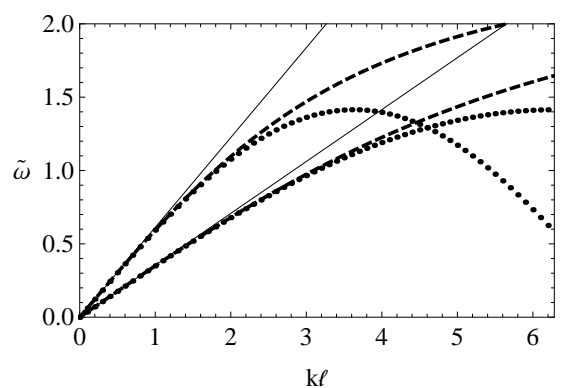

(b)

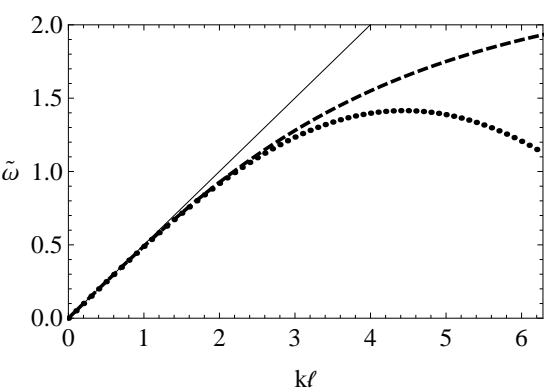

(c)

Figure 2: Comparison of dispersion curves for discrete system (dotted line), classical continuum approximation (solid line), higher-order continuum approximation (dashed line) for different direction of propagation $(\beta=1 / 30)$ : a) $\gamma=0$, b) $\gamma=\pi / 6$, c) $\gamma=\pi / 4$.

coefficients depend on only six constants $C_{i}$, thus it is necessary to introduce some simplification in the parameter selection.

An inspection of the higher order inertia terms suggests that to reproduce the expected symmetric response for propagation along $x$ and $y$, it is necessary to set $C_{1}=C_{5}, C_{2}=C_{4}$ and $C_{3}=$ $C_{6}$. Furthermore, we will retain all terms proportional to $\beta^{2}$ and those proportional to $\ell^{2}$, but not those proportional to $\beta^{2} \ell^{2}$. Equating to zero the remaining coefficients, the parameters that cancel the fourth-order derivatives are

$$
C_{1}=C_{2}=-\frac{1}{12} \quad C_{3}=\frac{1}{12}\left(1-\beta^{2}\right)
$$

Finally, the resulting continuum model given by

$$
\begin{aligned}
2 \rho \ddot{u}-\frac{1}{6} \rho \ell^{2} \ddot{u}_{x x} & =\frac{1}{2} E\left(2 u_{x x}+\beta^{2} u_{y y}+\beta^{2} v_{x y}\right) \\
2 \rho \ddot{v}-\frac{1}{6} \rho \ell^{2} \ddot{v}_{y y} & =\frac{1}{2} E\left(2 v_{y y}+\beta^{2} v_{x x}+\beta^{2} u_{x y}\right)
\end{aligned}
$$

possess only higher-order inertia terms.

In the following Section the dispersion properties of the proposed higher order continuum model will be investigated. The comparison with the dispersion curves of the lattice material will be used to asses the validity of the proposed approximation.

\section{Dispersion analysis}

The quality of a continuum approximation theory can be assessed by comparison of its dispersion relations with the ones of the discrete model. In the dispersion analysis, plane harmonic waves are considered and the components of the displacements field vector $\mathbf{q}_{m, n}=\left\{u_{m, n}, v_{m, n}, \theta_{m, m}\right\}^{\mathrm{T}}$ for the inner nodes have the form

$$
\begin{aligned}
& u_{m, n}(t)=A \exp \left[\mathrm{i}\left(\omega t-m k_{x} \ell-n k_{y} \ell\right)\right] \\
& v_{m, n}(t)=B \exp \left[\mathrm{i}\left(\omega t-m k_{x} \ell-n k_{y} \ell\right)\right] \\
& \theta_{m, n}(t)=C \exp \left[\mathrm{i}\left(\omega t-m k_{x} \ell-n k_{y} \ell\right)\right]
\end{aligned}
$$

where $A, B$ and $C$ are the wave amplitudes, $\mathrm{i}=\sqrt{-1}$ and $\omega$ is the angular frequency. In equations (15), $k_{x}=k \cos \gamma$ and $k_{y}=k \sin \gamma$ are the wavenumbers and $\gamma$ is the direction of propagation. Substituting equations (15) into the equations of motion (6) yields a set of three algebraic equations

$$
2\left[1+\beta^{2}-\cos \left(k_{x} \ell\right)-\beta^{2} \cos \left(k_{y} \ell\right)-\ell^{2} \frac{\rho}{E} \omega^{2}\right] A+\mathrm{i} \ell \beta^{2} \sin \left(k_{y} \ell\right) C=0
$$

$$
2\left[1+\beta^{2}-\beta^{2} \cos \left(k_{x} \ell\right)-\cos \left(k_{y} \ell\right)-\ell^{2} \frac{\rho}{E} \omega^{2}\right] B-\mathrm{i} \ell \beta^{2} \sin \left(k_{x} \ell\right) C=0
$$

$$
3 i \sin \left(k_{y} \ell\right) A-3 i \sin \left(k_{x} \ell\right) B-\ell\left[\cos \left(k_{x} \ell\right)+\cos \left(k_{y} \ell\right)+8\right] C=0
$$

Nontrivial solutions exist if and only if the determinant $\Delta\left(\omega, k_{x}, k_{y}\right)$ of the system (16) is zero. Thus, the dispersion relations are solutions of the equation $\Delta\left(\omega, k_{x}, k_{y}\right)=0$. The positive roots obtained numerically solving the characteristic equations (16) characterize the dispersion relations of compression and shear waves in the discrete system. The dispersion curves have been plotted in figure (2) for specific direction of propagation $\gamma$.

For the classical continuum and the continuum with higherorder inertia terms a similar set of equations can be obtained by substituting the harmonic solutions of the continuous form

$$
\begin{aligned}
& u(x, y ; t)=A \exp \left[\mathrm{i}\left(\omega t-k_{x} x-k_{y} y\right)\right] \\
& v(x, y ; t)=B \exp \left[\mathrm{i}\left(\omega t-k_{x} x-k_{y} y\right)\right]
\end{aligned}
$$

into the systems (9) and (14), respectively.

For the classical continuum one obtains the relation

$$
\tilde{\omega}=\frac{k \ell}{2 \sqrt{2}} \sqrt{2+\beta^{2} \pm \sqrt{2\left(1-\beta^{2}\right)[1+\cos (4 \gamma)]+\beta^{4}}}
$$

for compression and shear waves, where $\tilde{\omega}=\omega \sqrt{\rho \ell^{2} / E}$ is a non-dimensional frequency parameter. Equation (18) reveals that the classical continuum is not dispersive because the frequency $\omega$ is directly proportional to the wave number $k$.

For the higher order continuum, inserting equations (17) into 


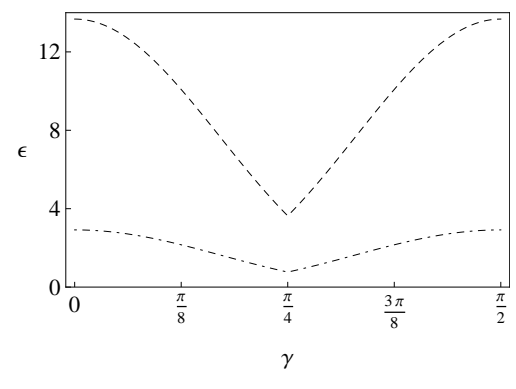

(a)

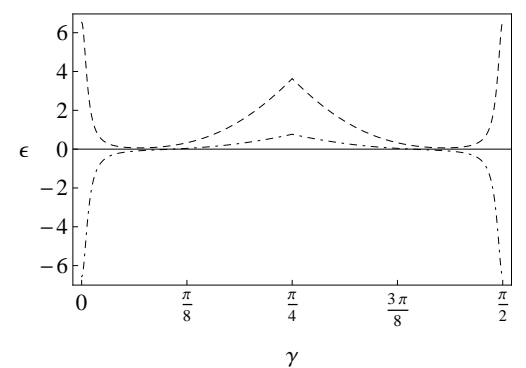

(b)

Figure 3: Relative approximation error against the direction of propagation for $k \ell=2$ (dot-dashed line) and $k \ell=3$ (dashed): a) compression waves; b) shear waves.

equations (14) yields a system of two algebraic equations

$$
\begin{aligned}
& {\left[3\left(2\left(k_{x} \ell\right)^{2}+\beta^{2}\left(k_{y} \ell\right)^{2}\right)-\left(12+\left(k_{x} \ell\right)^{2}\right) \tilde{\omega}^{2}\right] A} \\
& +\beta^{2}\left(k_{x} \ell\right)\left(k_{y} \ell\right)\left(3-\tilde{\omega}^{2}\right) B=0 \\
& \beta^{2}\left(k_{x} \ell\right)\left(k_{y} \ell\right)\left(3-\tilde{\omega}^{2}\right) A \\
& +\left[3\left(\beta^{2}\left(k_{x} \ell\right)^{2}+2\left(k_{y} \ell\right)^{2}\right)-\left(12+\left(k_{y} \ell\right)^{2}\right) \omega^{2}\right] B=0
\end{aligned}
$$

The results of the dispersion analysis for a lattice material with $\beta=1 / 30$ are illustrated in figure 2 , where the dispersion curves for discrete, classical, and higher-order models are shown by dotted, solid and dashed lines, respectively. Frequency $\tilde{\omega}$ and wavenumbers $k \ell$ are presented in dimensionless form, for different directions of propagation $\gamma$. Both the compressional and shear branches evaluated from the classical and higher-order continuum approximations are tangent to the dispersion curves of the discrete system at the long-wave limit $k \ell \rightarrow 0$. Further, in the region of wave lengths near the limit $k \ell \rightarrow 0$, the two branches of the model with higher-order inertia are in good agreement with the ones of the discrete system. For the higher normalised wave numbers the continuum approximation starts to deviate. However, the two dispersion branches are numerically stable for all the direction $\gamma$ and the higher-order model describes the dispersion of waves, in any case overlooked by classical elasticity theory, in a wide region of wave numbers. The accuracy of the proposed approximation can be estimated by considering the relative error $\epsilon$ plotted in figure 3 for two fixed value of $k \ell$ and a full range of direction of propagation.

\section{Concluding remarks}

This work investigates the use of higher-order gradient continuum models to simulate wave dispersion in materials with periodic lattice microstructure. The equations of motion for an equivalent continuum approximating the actual material have been derived combining the FE discretisation of the UC with the continualisation approach based on the Taylor series expansion of the variable fields. The technique applied here to simple periodic square lattice can be easily generalised and implemented in a FE code for analysis of complex periodic lattice structure.
From the application of the continualisation approach, it has been shown that the continuum models enriched with higherorder gradient terms introduce in the analysis the characteristic length parameter characterizing the microstructure. Specifically, a continuum model enriched with higher-order inertia terms has been proposed as alternative to the continuum description obtained with classical elasticity theory.

After deriving the continuum equations of motion, the dispersion analysis has been elaborated. The comparison of the dispersion curves of the lattice with the dispersion curves for the classical and the enhanced continua reveals that the gradient enriched model does not generate numerical instability and it is able to capture the wave dispersion properties missed with classical continuum approximation.

\section{Acknowledgments}

The authors gratefully acknowledge the financial support of the Defence Science and Technology Laboratory (DSTL), contract number DSTLX-1000024926.

\section{References}

[1] L. J. Gibson, F. Ashby, Cellular solids: structures and properties, Cambridge University Press, Cambridge, UK, 2001.

[2] M. Ruzzene, F. Scarpa, Directional and band-gap behavior of periodic auxetic lattices, Physica Status Solidi (b) 242 (2005) 665-680.

[3] A. Shrikantha Phani, J. Woodhouse, N. A. Fleck, Wave propagation in two-dimensional periodic lattices, Journal of Acoustical Society in America 119 (2006) 1995-2005.

[4] M. Ostoja-Starzewki, Lattice models in micromechanics, Applied Mechanics Reviews 55 (2002) 35-60.

[5] S. Gonella, M. Ruzzene, Homogenization and equivalent in-plane properties of two-dimensional periodic lattices, International Journal of Solids and Structures 45 (2008) 2897-2915.

[6] A. A. Vasiliev, S. V. Dmitriev, Y. Ishibashi, T. Shigenari, Elastic properties of a two-dimensional model of crystals containing particles with rotational degrees of freedom, Physical Review B 65 (2002) 65-71.

[7] I. S. Pavlov, A. I. Potapov, G. A. Maugin, A 2d granular medium with rotating particles, International Journal of Solids and Structures 43 (2006) 6194-6207.

[8] A. A. Vasiliev, S. V. Dmitriev, A. E. Miroshnichenko, Multi-field approach in mechanics of structural solids, International Journal of Solids and Structures 47 (2010) 510-525.

[9] M. Lombardo, H. Askes, Elastic wave dispersion in microstructured membranes, Proceedings of the Royal Society A (2010) 1789-1807.

[10] A. V. Pichugin, A. Askes, A. Tyas, Asymptotic equivalence of homogenization procedures and fine-tuning of continuum-theories 313 (2008) 858-874. 